\title{
Update on Food Allergy
}

\author{
Rachel Peters (N/A) ${ }^{1}$, Marta Krawiec ${ }^{2}$, Jennifer Koplin ${ }^{1}$, and Alexandra Santos ${ }^{2}$ \\ ${ }^{1}$ Murdoch Childrens Research Institute \\ ${ }^{2}$ King's College London
}

September 4, 2020

\begin{abstract}
Food allergy is a major public health issue with growing prevalence in the urbanized world and significant impact on the lives of allergic patients and their families. Research into the risk factors that have contributed to this increase and their underlying immune mechanisms could lead us to definitive ways for treatment and prevention of food allergy. For the time being, introduction of peanut and other allergenic foods in the diet at the time of weaning seems to be an effective way to prevent the development of food allergy. Improved diagnosis and appropriate management and support of food allergic patients are central to patient care with food immunotherapy and biologicals making the transition to clinical practice. With the new available treatments it is becoming increasingly important to include patient's and family preferences to provide a management plan tailored to their needs.
\end{abstract}

\section{Hosted file}

Review article on Food Allergy for PAI FINAL.DOCX available at https://authorea.com/users/ 356334/articles/479245-update-on-food-allergy 